\title{
Barriles de absorción y contención del impacto: reducción de mortalidad por accidentes de tránsito
}

\author{
Carlos Arreola-Rissa, MD,(1) Jesús Santos-Guzmán, MS, (1) Adol Esquivel-Guzmán, MD, MS, (2) \\ Charles Mock, MD, PhD, FACS, ${ }^{(3)}$ Alejandro Herrera-Escamilla, Lic.(1)
}

\section{Arreola-Rissa C, Santos-Guzmán J, Esquivel-Guzmán A, Mock C, Herrera-Escamilla A. Barriles de absorción y contención del impacto: reducción de mortalidad por accidentes de tránsito. Salud Publica Mex 2008;50 supl I:S55-S59.}

\section{Resumen}

Objetivo. Determinar el efecto en la reducción de la mortalidad por accidentes de tránsito de la instalación de un dispositivo de absorción y contención de impacto (BAFI), en cruceros de eje estrecho en la ciudad de Monterrey, México. Material y métodos. Se obtuvo información sobre accidentes del I de enero de 2000 al 3 I de diciembre de 2005 en cruceros con o sin BAFI. Resultados. En el periodo de estudio se registraron 142 accidentes en cruceros de eje estrecho, con 59 muertes en cruceros sin BAFI y cinco en aquellos donde se instaló este dispositivo. Conclusiones. Los dispositivos BAFI representan una medida rápida y de bajo costo para reducir la mortalidad y gravedad de lesiones por accidentes en cruceros de eje estrecho.

Palabras clave: barril de absorción y contención de impacto (BAFI); cruceros de eje estrecho; mortalidad; accidentes de tránsito; México

\section{Arreola-Rissa C, Santos-Guzmán J, Esquivel-Guzmán A,} Mock C, Herrera-Escamilla A.

Impact-absorbing and containment barriers:

reduction in accident mortality.

Salud Publica Mex 2008;50 suppl I:S55-S59.

\begin{abstract}
Objective. To determine the effect of the installation of an impact absorption and containment device (Spanish abbreviation, BAFI) on the reduction of traffic accident fatalities at edge crossings in the city of Monterrey, Mexico. Materials and Methods. Information about accidents was obtained from January I, 2000 to December 3I, 2005 from intersections with and without BAFI. Results. During the study period, 142 accidents were registered in edge intersections, with 59 deaths in intersections without BAFls and five in those where the devices were installed. Conclusions. The BAFI devices represent a quick and low-cost step for reducing mortality and serious injury due to accidents in edge crossings.
\end{abstract}

Key words: impact-absorbing barriers; highway edge crossing; mortality; traffic accidents; Mexico

(I) Escuela de Medicina, Instituto Tecnológico y de Estudios Superiores de Monterrey. Nuevo León, México.

(2) School of Health Information Sciences, The University of Texas Health Science Center at Houston. Houston, Texas, USA.

(3) Violence and Injury Prevention and Disability Department, World Health Organization. Geneva, Switzerland. 
$E^{1}$ desarrollo urbano de cualquier ciudad supone establecer o mejorar vías de comunicación eficientes; el trazo de estas vialidades implica la posibilidad de recibir mayor cantidad de vehículos, la rapidez del desplazamiento, las conexiones en sitios específicos que acorten distancias y la seguridad de los tripulantes de los vehículos. La movilización de flotillas vehiculares en mayor volumen y velocidad exige nuevos estándares para las carreteras y vehículos que le aseguren al conductor menores riesgos. Se han incorporado varias medidas posibles para mejorar la velocidad de traslado y la seguridad de los conductores. Los ingenieros automotrices han incluido elementos de protección: cinturones de seguridad, bolsas autoinflables, reforzamiento de la cabina de los pasajeros, motores y eje de la dirección colapsables, paneles de vidrios antiastillables, accesorios que no protruyen hacia la cabina, reforzamiento de la carrocería con paneles de absorción de fuerza de impacto, defensas y parachoques reforzados y mejores frenos. A su vez, muchos cuerpos legislativos y autoridades competentes han promovido leyes que regulan el uso del cinturón de seguridad, consumo de alcohol, distractores del conductor como el uso de teléfonos celulares mientras se conduce y capacitación vial a los conductores. Por último, las autoridades locales y nacionales han desarrollado planes de desarrollo urbano que incluyen mejores señalamientos, límites de velocidad, uso de radares o cámaras automáticas, campanas de detección de conductores alcoholizados, promoción de uso del cinturón, y estructuras de contención y absorción de fuerza de impacto como rieles o barras, violetas, ranuración del asfalto y otros más.

Algunos estudios señalan que los embotellamientos de tránsito (con su natural disminución de la velocidad de los vehículos) son uno de los factores que reducen en grado considerable el riesgo de morir en accidentes viales. ${ }^{1}$ Aunque uno de los efectos es bueno, entorpecer la circulación de los automóviles también representa problemas y costos secundarios. La mayoría de las autoridades busca construir escenarios de velocidad razonable de traslado con el menor riesgo posible. En la ciudad de Monterrey, la construcción de vías rápidas ha mostrado una tendencia creciente, desde los pasos a desnivel hasta las vías elevadas con puentes. Muchas de estas vías rápidas incluyen rampas, divisores viales, carriles de entrada o salida, así como carriles convergentes, y exigen del conductor una decisión rápida. Como efecto, existe un riesgo relativo de impacto si el conductor no decide a tiempo si realiza cambios de carril con brusquedad o sin señalización, o bien, si frena de modo intempestivo. Se han diseñado varios dispositivos que sirven al mismo tiempo de señalización y tienen la capacidad de absorber el impacto. Algunos de estos dispositivos son complejos y elaborados y por lo tanto costosos (figura 1).

Otra alternativa ha sido la utilización de los barriles de absorción y contención del impacto (BAFI), que son receptáculos de plástico reforzado capaces de alojar $180 \mathrm{~L}$ de agua y que se colocan alineados en número de dos a cuatro y se fijan mediante un cable especial. Se considera que los BAFI pueden reducir la velocidad del vehículo hasta en 30 a 50 por ciento. El costo de uno de estos atenuadores es de unos 150 pesos (15 dólares) y su mantenimiento es muy económico y requiere transportación, relleno de agua o reposición en caso de daño (en un accidente vial el costo se le carga al conductor del vehículo accidentado). ${ }^{2}$

Desde hace más de cinco años se ha operado en la ciudad de Monterrey un programa de instalación y mantenimiento de barriles atenuadores del impacto, como una alternativa para extender la seguridad en los cruceros de eje fijo. Es el propósito del presente estudio

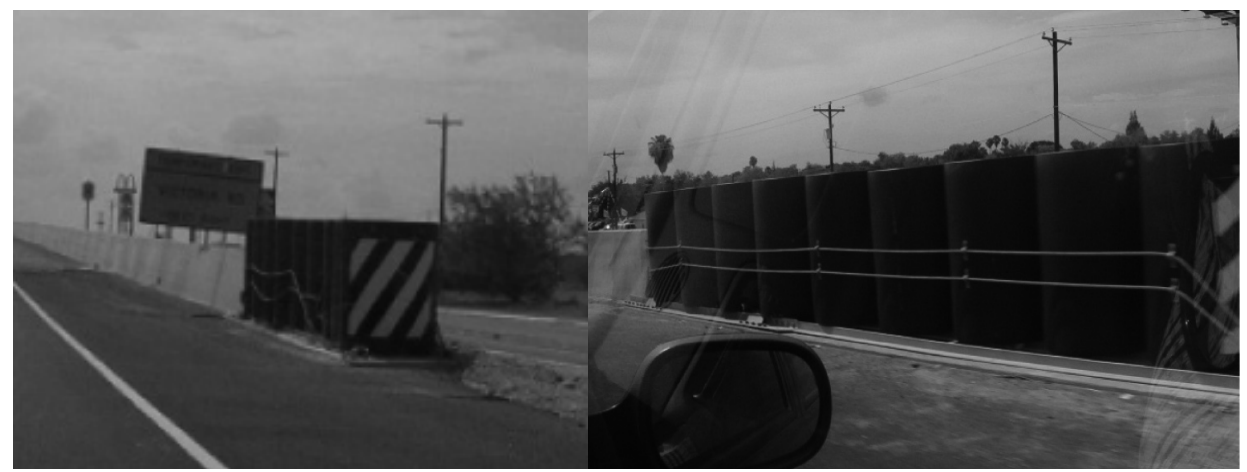

Figura I.Atenuadores de impacto de acero usados en el estado de Texas, Estados Unidos 
mostrar el efecto de la disminución de la mortalidad en accidentes de tránsito como consecuencia de la colocación de estos BAFI en cruceros de eje fijo.

\section{Material y métodos}

Durante 2001 el municipio de Monterrey inició un programa de prevención de mortalidad en cruceros con eje estrecho (bifurcación de la calle, con un divisor de eje estrecho o triangular), gracias al cual se han instalado 250 BAFI cada año en 45 cruceros, la mayor parte en nuevos cruceros y algunos como reposición de los dañados (figuras 2 y 3 ). Las configuraciones de los barriles varían según sea la disposición del crucero: lineal, triangular o romboide. El número de atenuadores y su configuración dependen del tipo de crucero y las velocidades esperadas de los vehículos circulantes. Otro criterio para la instalación de nuevos BAFI es la

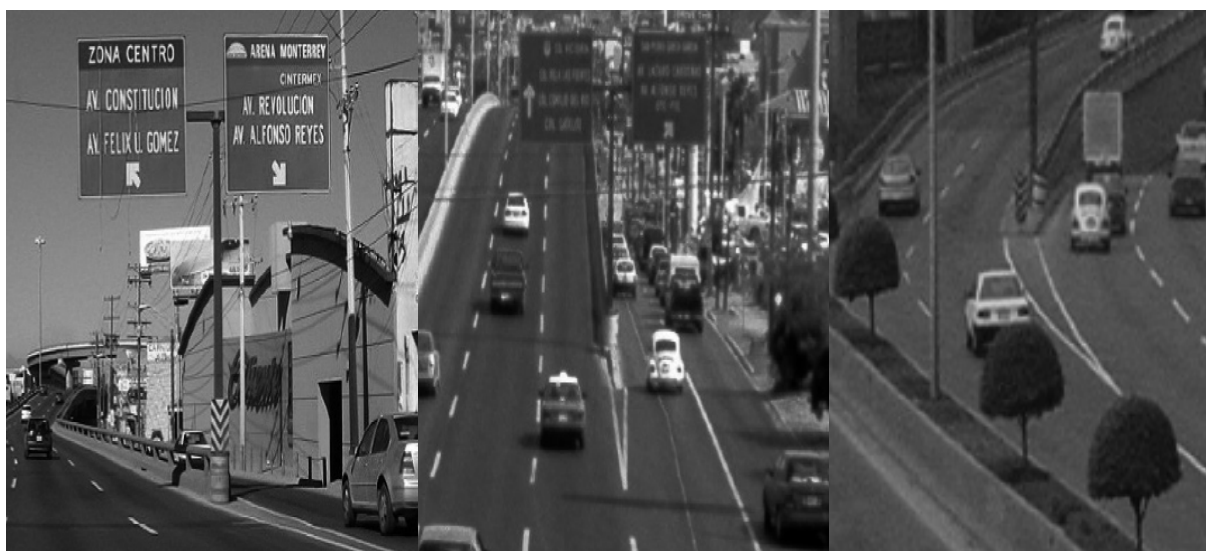

Figura 2. EJEMPLOS DEL BARRIL de ABSORCIÓN Y CONTENCIÓN DEL IMPACTO (BAFI)

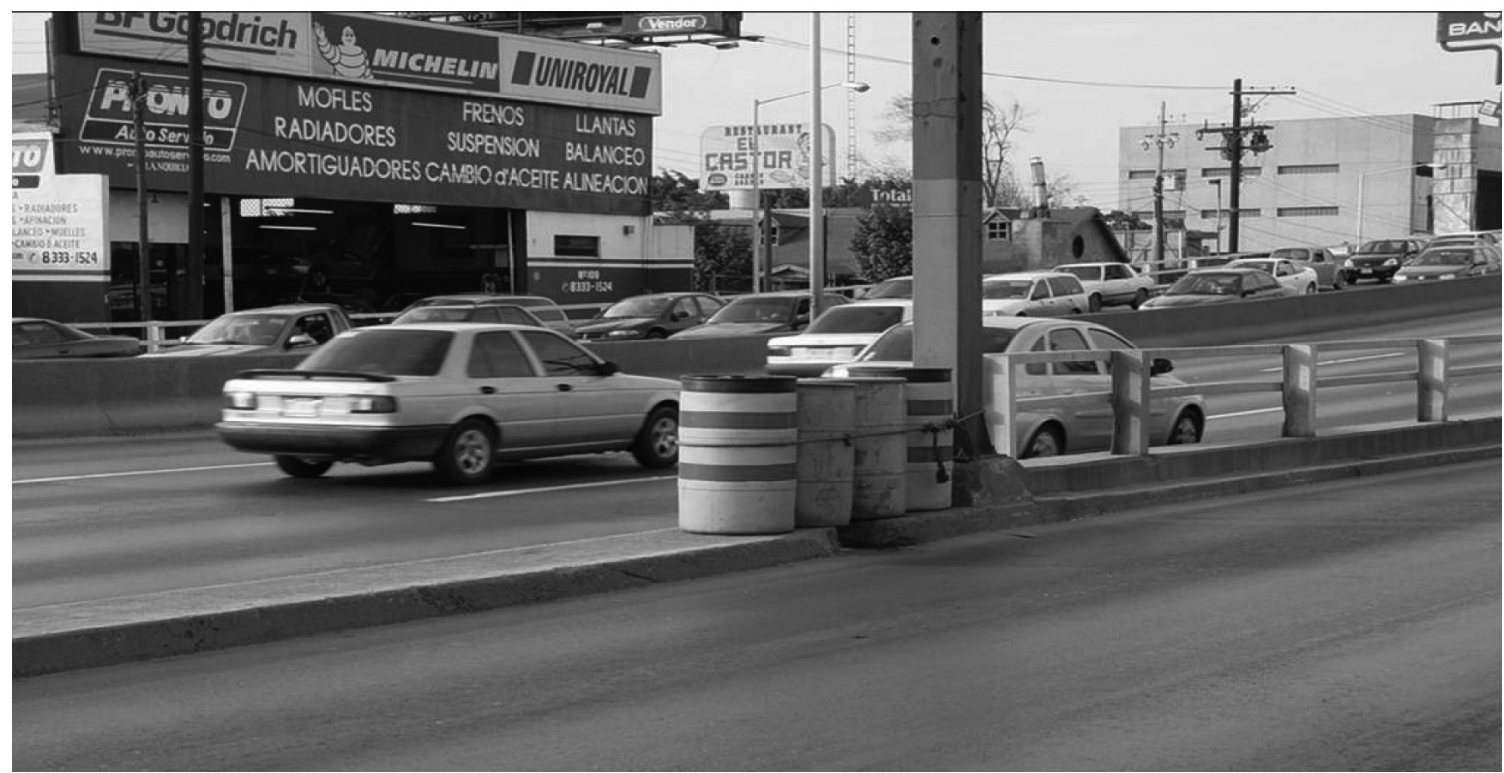

FiguRA 3. EJEMPLOS DE BARRILES de ABSORCIÓN Y CONTENCIÓN DEL IMPACTO (BAFI) EN LOS QUE SE OBSERVA SU COLOCACIÓN Y SUJECIÓN 
cantidad de accidentes ocurridos en dichos cruceros, sobre todo los fatales.

Del 1 de enero de 2000 al 31 de diciembre de 2005 se obtuvo información del número de accidentes en cruceros de eje fijo del municipio de Monterrey, incluidos el crucero específico, el número de accidentes, lesionados y muertos, la fecha del accidente y la instalación o ausencia de BAFI. La información se obtuvo de la Dirección de Vialidad y Tránsito de Monterrey. La información se consideró suficiente y no se verificó en otras fuentes. No se analizaron otras variables, como el uso de cinturón de seguridad ni la velocidad calculada al impacto de cada choque, por la inconsistencia de su notificación en los archivos oficiales.

\section{Resultados}

De 2000 a 2005 se presentaron 150454 accidentes (fatales y no fatales; alrededor de $9 \%$ se relacionó con objetos fijos) (cuadro I). La tasa de mortalidad por 100000 habitantes del total de accidentes fue de $0.86 \pm 0.4$ (límites, 0.5-1.63) y para los accidentes con objetos fijos de $0.35 \pm$ 0.18 (límites, 0.17-0.61) (cuadros I y II).

En el cuadro II se puede observar un aumento constante de la población de Nuevo León y de la flota vehicular, mientras que los accidentes de tráfico siguen una tendencia opuesta. El número de accidentes contra objetos fijos representa $9 \pm 1.8 \%$ (límites, 8.1-12.2\%) del total de accidentes y tiene una tendencia de crecimiento lento, similar a la del total de accidentes (fatales y no fatales). En el periodo del estudio se presentaron $1.4 \pm$ 0.45 muertes por cada 1000 accidentes, $0.6 \pm 0.2$ muertes en cruceros estrechos sin BAFI por cada 1000 accidentes y $0.03 \pm 0.01$ muertes en cruceros de eje estrecho con BAFI por cada 1000 accidentes (véase el cuadro III). En el cuadro III se puede observar que en los últimos cinco años se ha registrado una tendencia constante, sin cambios significativos, con excepción del 2005, en el número de muertes vinculadas con accidentes de tránsito en ocupantes de vehículo, con un promedio de $35.2 \pm 17.4$ muertes por año (límites, 20-69). De éstas, se reconoció un promedio de $14.2 \pm 7.5$ (límites, 7-26) de accidentes fatales contra objetos fijos sin BAFI y 0.8 (límites, 0-5) decesos por año en crucero de eje estrecho y BAFI (cuadro III).

\section{Discusión}

Algunos investigadores señalan que el factor humano representa más de dos tercios de las causas de accidentes, las condiciones de la carretera menos de un tercio y las condiciones del vehículo cerca de $10 \%$ de los casos. ${ }^{3}$ Es por ello que una de las prioridades de las autoridades para disminuir la mortalidad por accidentes de tránsito es la educación y supervisión policial de los conductores / pasajeros, y como segundo punto figura el

Cuadro I

Accidentes de tránsito fatales y no fatales en el municipio de Monterrey. Nuevo León, México, 2000-2005

\begin{tabular}{lrrrrrrrr} 
Año & 2000 & 2001 & 2002 & 2003 & 2004 & 2005 & Total \\
Todos & $1537 \mid$ & 28198 & 29123 & 29492 & 24688 & 23582 & 150454 \\
\hline $\begin{array}{l}\text { Cruceros con eje estrecho } \\
(\%)\end{array}$ & 1876 & 2514 & 2456 & $277 \mid$ & 2007 & 2106 & 13730 \\
(12 & 9 & 8 & 9 & 8 & 9 & 9
\end{tabular}

Fuente: Secretaría de Vialidad y Tránsito de Monterrey y Conapo

Cuadro II

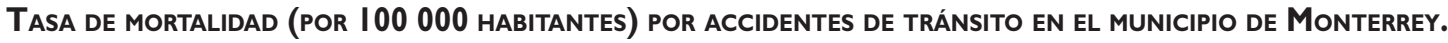
Nuevo León, MéXıco, 2000-2005

\begin{tabular}{|c|c|c|c|c|c|c|c|}
\hline Año & 2000 & 2001 & 2002 & 2003 & 2004 & 2005 & Promedio \\
\hline Total & 0.83 & 0.5 & 0.89 & 0.68 & 0.62 & 1.63 & $0.9 \pm 0.4$ \\
\hline Cruceros con eje estrecho & 0.26 & 0.2 & 0.49 & 0.34 & 0.17 & 0.61 & $0.3 \pm 0.17$ \\
\hline Población Monterrey (CONAPO) & I 124339 & 1128379 & $1|3| 863$ & I 134834 & I 137334 & 1139417 & I 132694 \\
\hline
\end{tabular}

Fuente: Secretaría de Vialidad y Tránsito de Monterrey y Conapo 


\section{Cuadro III \\ ACCIDENTES DE TRÁNSITO FATALES Y USO DE BARRILES DE ABSORCIÓN Y CONTENCIÓN DEL IMPACTO (BAFI) en el municipio de Monterrey, Nuevo León, México}

\begin{tabular}{lrrrrrrrr} 
Año & 2000 & 2001 & 2002 & 2003 & 2004 & 2005 & Total & Promedio \\
& & & & & & & & \\
Total & 32 & 20 & 36 & 28 & 26 & 69 & 211 & $35.2 \pm 17.4$ \\
\hline Muertes en cruceros con eje estrecho & 10 & 8 & 20 & 14 & 7 & 26 & 85 & $14.2 \pm 7.5$ \\
\hline Muertes con BAFI & 2 & 1 & 0 & 0 & 0 & 2 & 5 & $0.8 \pm 0.9$
\end{tabular}

Fuente: Secretaría de Vialidad y Tránsito de Monterrey y Conapo

establecimiento de mejores condiciones de las carreteras y vías públicas. Aunque el efecto de las vías rápidas con tránsito lento redunde en menor mortalidad por accidentes de tráfico, en una ciudad moderna deben buscarse alternativas con vialidades fluidas y rápidas, pero con bajo riesgo de mortalidad. Cabe agregar que al aumentar la velocidad de los automóviles y camiones se le agrega riesgo a los que transitan a pie o se desplazan en bicicletas o motocicletas. Para solucionar este problema es preciso analizar programas de educación vial y limitar ciertos puntos de las carreteras al acceso exclusivo de vehículos de motor o establecer franjas laterales de emergencia para que transiten los peatones. El presente estudio muestra que la utilización de los BAFI contribuye a disminuir la mortalidad al servir como indicador visual del cruce y tener la capacidad de absorber y contener la fuerza de impacto. En el periodo de estudio, sólo en cinco casos se presentaron accidentes fatales en presencia de los barriles y se consideró que la fuerza del impacto fue de tal magnitud que aun con los barriles se produjo la muerte de los ocupantes del vehículo. En cuanto a la aparente elevación de los casos en 2005 en relación con los años anteriores, se consideró como posible explicación el efecto de las temporadas de lluvia más intensas y frecuentes y el deterioro de la carpeta asfáltica. En muchos tramos de las zonas estudiadas se iniciaron labores de reencarpetamiento en 2006 por considerar acentuado el deterioro y también por el incremento de los accidentes.

Los BAFI representan un elemento útil, económico y ampliamente disponible para reducir la mortalidad por accidentes en cruceros de eje fijo. Aunque existen muchos otros artefactos con mayor capacidad de absorción de impacto, los barriles descritos tienen una mayor versatilidad, con poca afectación de la estética de la estructura, un menor costo y una eficacia razonable en la prevención de muertes por accidentes de tránsito. Estos BAFI representan una opción conveniente en términos del costo para los programas de prevención de accidentes de países con economías en desarrollo.

\section{Conclusiones}

Debe considerarse que los BAFI son una medida útil, práctica y económicamente costeable para la prevención de muertes por choques de vehículos contra objetos fijos. La información usada en el artículo es preliminar y requiere un análisis posterior de un mayor número de casos para acumular mayor información y sustentar su utilización.

\section{Agradecimientos}

Los autores expresan su agradecimiento al Lic. Felipe de Jesús Cantú y al Lic. Ricardo Canavati Tafich, alcaldes de Monterrey en los periodos comprendidos del estudio, por el apoyo brindado para la realización del estudio, así como al Ing. Alejandro Castillo, de Planeación Urbana de la Secretaría de Vialidad y Tránsito de Monterrey.

\section{Referencias}

I. Richter ED, Barach P, Ben-Michael E, Berman T. Death and injury from motor vehicle crashes:A public health failure, not an achievement. Inj Prev 200।;7:176-178.

2.Arreola-Rissa C, Herrera-Escamilla AJ, de la Cruz O, Mock CN.Análisis de la mortalidad por accidentes viales en la ciudad de Monterrey. Qué hemos aprendido y estrategias a implementar. Trauma 2003;6:4-I4.

3. Montoso-González L. Estrategias para la prevención de los accidentes de tráfico. La prevención de la seguridad vial. Ponencia desarrollada en el II Congreso Internacional de Tráfico Urbano. Disponible en: http://www. forcupol.com/s-vial.htm. 